\title{
Classification of broadband echoes from prey of a foraging Blainville's beaked whale
}

\author{
Benjamin A. Jones, ${ }^{\text {a) }}$ Timothy K. Stanton, Andone C. Lavery, and Mark P. Johnson \\ Department of Applied Ocean Physics and Engineering, Woods Hole Oceanographic Institution, \\ Woods Hole, Massachusetts 02543-1053 \\ Peter T. Madsen \\ Department of Biological Sciences-Zoophysiology, University of Aarhus, Denmark \\ Peter L. Tyack \\ Department of Biology, Woods Hole Oceanographic Institution, Woods Hole, Massachusetts 02543-1053
}

(Received 5 September 2007; revised 22 November 2007; accepted 4 December 2007)

\begin{abstract}
Blainville's beaked whales (Mesoplodon densirostris) use broadband, ultrasonic echolocation signals with a $-10 \mathrm{~dB}$ bandwidth from 26 to $51 \mathrm{kHz}$ to search for, localize, and approach prey that generally consist of mid-water and deep-water fishes and squid. Although it is well known that the spectral characteristics of broadband echoes from marine organisms vary as a function of size, shape, orientation, and anatomical group, there is little evidence as to whether or not free-ranging toothed whales use spectral cues in discriminating between prey and nonprey. In order to study the prey-classification process, a stereo acoustic tag was deployed on a Blainville's beaked whale so that emitted clicks and the corresponding echoes from targets in the water could be recorded. A comparison of echoes from targets apparently selected by the whale and those from a sample of scatterers that were not selected suggests that spectral features of the echoes, target strengths, or both may have been used by the whale to discriminate between echoes. Specifically, the whale appears to favor targets with one or more nulls in the echo spectra and to seek prey with higher target strengths at deeper depths. (C) 2008 Acoustical Society of America. [DOI: 10.1121/1.2828210]
\end{abstract}

PACS number(s): 43.80.Lb, 43.30.Sf, 43.30.Pc, 43.80.Ev [WWA] Pages: 1753-1762

\section{INTRODUCTION}

Toothed whales emit broadband, ultrasonic signals to navigate and locate prey (Au, 1993). Although these uses of sound have proven difficult to study in the wild, considerable progress has been made with various species of dolphins in captivity. It has been shown that trained dolphins can discriminate between targets with subtle differences in size, shape, and material composition using information contained in the broadband echoes (Nachtigall, 1980). Identifying the specific features of acoustic signals that dolphins use to discriminate between targets is the subject of ongoing research. Findings thus far have led to the speculation that dolphins use spectral information to discriminate between artificial objects (Vel'min and Dubrovskiy, 1976; Dubrovskiy, 1989; Au and Pawloski, 1989; Fuzessery et al., 2004).

In independent studies, research on acoustic scattering from individual marine organisms has shown that different anatomical groups of animals can be classified by the frequency spectra of their backscattered signals (Martin et al., 1996; Stanton et al., 1998; Martin Traykovski et al., 1998). One goal of this research has been to develop acoustic classification schemes based on the frequency spectra of the scattered signals. Constructive and destructive interference of the sound wave, scattered by anatomical features such as tissue

\footnotetext{
a) Author to whom correspondence should be addressed. Electronic mail: ben.jones@navy.mil; current affiliation: U.S. Naval Maritime Forecast Center/Joint Typhoon Warning Center, Pearl Harbor, HI 96860.
}

interfaces, gas inclusions, and bone, create a frequencydependent interference pattern. This pattern is specific to the size, shape, orientation, material properties (i.e. sound speed and density), and internal morphology of the scatterer (review by Simmonds and Maclennan, 2005). Studies of broadband acoustic scattering from individual zooplankton (e.g., Stanton et al., 1998) and fish (e.g., Reeder et al., 2004) have shown that structure in the spectra of high frequency, acoustic backscattering is, indeed, due to anatomical features and is highly affected by orientation. In addition, active broadband acoustic systems have been used in efforts to achieve reliable species recognition based on the spectral signature of backscatter from both individual fish (Au and Benoit-Bird, 2003) and aggregations of fish (Simmonds et al., 1996; Zakharia et al., 1996).

These findings, combined with the evidence for spectral selection in captive dolphins, suggests that it is possible that free-ranging toothed whales use spectral features of broadband, acoustic backscattering to aid in the classification of prey. A few studies have shown prey selection by toothed whales in the wild (MacLeod et al., 2006; Whitehead et al., 2003), but the basis for such selection is not known. Observing acoustically based prey selection in free-ranging toothed whales poses a significant challenge: while sounds emitted by echolocating whales can be recorded with surfacedeployed hydrophones, the lower amplitude echoes, scattered by prey, are considerably more difficult to measure. Recently, this problem has been overcome, for some species of beaked whales, by using acoustic recording tags (DTAGs) 
affixed directly to echolocating whales (Johnson and Tyack, 2003; Zimmer et al., 2005; Madsen et al., 2005; Johnson et al., 2006). Using these devices, both the whale's emitted signals and echoes from scatterers in the water column are recorded, providing a detailed view of the echolocation process in the wild.

In one such study, Madsen et al., (2005) reported data from a DTAG attached to a Mesoplodon densirostris. This species of beaked whale uses broadband signals, or clicks, to search for and localize prey consisting of mesopelagic fishes and squid (Johnson et al., 2004; Pauly et al., 1998). Numerous echoes from scatterers in the water column were recorded including some that were associated with series of rapid clicks, called buzzes, emitted by the whale. These distinctive sounds, also observed in other toothed whales and bats, have been associated with prey capture attempts (Madsen et al., 2002; Miller et al., 2004; Griffin, 1958; Johnson et al., 2006). Although the whale ensonified a large number of scatterers on each dive, it selected only a small percentage of them for capture. This suggests that the animal is actively selecting certain types of prey (Madsen et al., 2005); however, the criteria for such selection were not examined in that study.

In this paper a data set from a related study (Johnson et al., 2006) is examined in order to compare the acoustic scattering signatures of the targets selected by the whale with those not selected. Both the spectral characteristics and relative target strengths of individually resolved echoes are examined. Significant differences were observed with respect to spectral content of selected and nonselected targets. Moreover, differences in the echoes from prey selected from two different depth strata suggest that at least two distinct prey types may be targeted by the whale. Finally, the results are qualitatively compared with previous laboratory and modeling studies of broadband scattering from marine organisms to provide context for echo-to-echo variation.

\section{MATERIALS AND METHODS}

\section{A. Signals emitted by the Blainville's beaked whale}

M. densirostris emit two different signals associated with different phases of echolocation-based foraging (Johnson et al., 2004, 2006). Distinctive frequency modulated (FM) clicks are produced at inter-click intervals (ICIs) of approximately $0.4 \mathrm{~s}$ while the whale searches and approaches prey. When the whale closes on a selected prey, it emits a rapid series of unmodulated clicks called a buzz. These clicks can be readily distinguished from the slower FM clicks by a lower output level and an ICI well below $0.1 \mathrm{~s}$ (Madsen et al., 2005). The FM clicks are relatively long with a duration of about $270 \mu$ s (Fig. 1(a)). The frequency spectrum of these clicks is within $10 \mathrm{~dB}$ of the maximum level between approximately 26 and $51 \mathrm{kHz}$ with very little energy at lower frequencies (Johnson et al., 2006). When observed close to the acoustic axis, FM clicks appear to have a consistently broad and relatively featureless spectrum, i.e., free of peaks and nulls (Fig. 1(b)), while off-axis clicks, generally, have more complex spectra (Johnson et al., 2006). Although the beamwidth of the M. densirostris FM
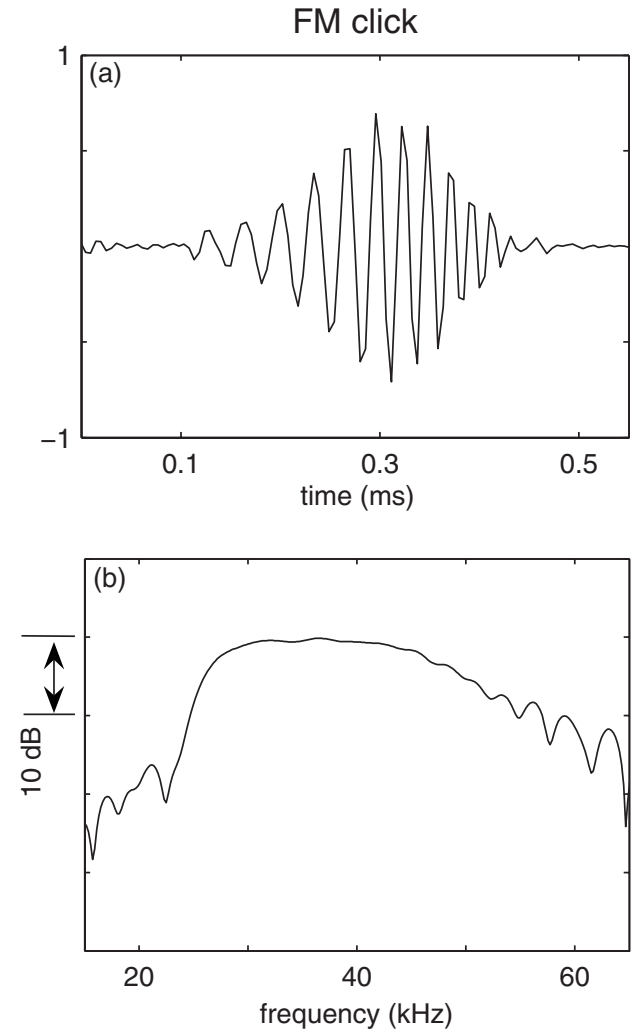

FIG. 1. Echolocation signal (FM click) of $M$. densirostris, observed during this study. The whale recorded was a conspecific foraging with the whale discussed in this study. (a) Time series (arbitrary vertical scale), (b) frequency spectrum.

clicks is not known, Zimmer et al. (2005) has estimated the $-3 \mathrm{~dB}$ beam width of another larger beaked whale with similar click characteristics, Ziphius cavirostris, at about $6^{\circ}$.

Although echoes from scatterers ensonified by FM clicks are frequently detectable in tag acoustic recordings, significantly fewer echoes are observed during buzzes. This is likely due to the lower output level of buzz clicks (Madsen et al., 2005; Johnson et al., 2006). For this reason, only echoes from targets ensonified by FM clicks are considered in the spectral and target strength analyses presented in this paper. Buzzes are used as an indication that the whale has selected a prey item and is approaching to capture it (Madsen et al., 2005).

\section{B. Data acquisition}

\section{Instrumentation}

Data reported here were collected with a miniature acoustic and orientation recording tag, the DTAG (Johnson and Tyack, 2003). The tag attaches to the surface of a whale with four suction cups and automatically releases after a programmed period of time. The DTAG contains two hydrophones spaced $2.5 \mathrm{~cm}$ apart which are sampled synchronously at a rate of $192 \mathrm{kHz}$ per channel. The hydrophones were calibrated in a pressure test facility to a depth of $500 \mathrm{~m}$. The overall frequency response is flat within $3 \mathrm{~dB}$ between 0.5 and $67 \mathrm{kHz}$ with the upper cutoff resulting from an antialias filter in the tag. The tag also samples a three-axis ac- 
celerometer and a three-axis magnetometer at $50 \mathrm{~Hz}$ for measuring orientation, and a pressure sensor for extracting depth.

\section{Field measurements}

An adult $M$. densirostris, tagged in October of 2004 near the Canary Islands, provided $9.5 \mathrm{~h}$ of acoustic data. The position of the tag during the dives was determined using photo documentation of the whale when surfacing between dives. The tag was initially placed on the right side of the whale but slid to a dorsal position approximately $1 \mathrm{~m}$ posterior of the blowhole after about $1 \mathrm{~h}$. This position minimized shading of the hydrophones by the whale's body providing favorable conditions to record echoes from scatterers ensonified by the whale. The tag remained near the dorsal ridge for the following three foraging dives. It is the data recorded during these dives that are examined in this study.

\section{Analysis methods}

Use of the whale's biosonar as a sound source and the animal's body as the platform for the receiver introduces several complexities in studying acoustic backscattering. First, it is not possible to make far-field measurements of the sounds emitted by the whale on which the tag is mounted. This means that the level and spectrum of the outgoing signal are not precisely known. In order to estimate the frequency response of scatterers, we restrict the analysis to the frequency range over which echolocation signals, recorded from other conspecifics, have been observed to be essentially featureless (Johnson et al., 2006). Furthermore, to estimate the relative target strengths of scatterers we use the near field recording of the whale's emitted signal as a proxy for output level and compensate for click to click variation in the amplitude of the emitted signal. A second complexity is that the position of the scatterer with respect to the whale's sonar beam can have a significant influence on spectral content. In order to reduce uncertainties associated with this effect, only echoes with echo-to-noise ratio (ENR) above $15 \mathrm{~dB}$ are chosen. Since scatterers located near the axis of the sonar beam will tend to produce the highest echo levels, selecting high ENR echoes ensures that a preponderance of the echoes examined are nearly on axis.

The approach taken here is to identify series of echoes associated with FM clicks, that appear to represent individual targets. Several parameters are computed for each echo (e.g., the number of nulls in the echo spectrum and the relative target strength) in the series. The parameters of the echoes in each series of echoes, or echo train, are compiled into a single statistic which can then be compared across trains. The objective is to determine if there are consistent differences in the parameters of echoes from targets apparently selected by the whale as compared to those not selected. Variation in the echo parameters will also be related to the depth and the overall density of acoustic scatterers in the environment.
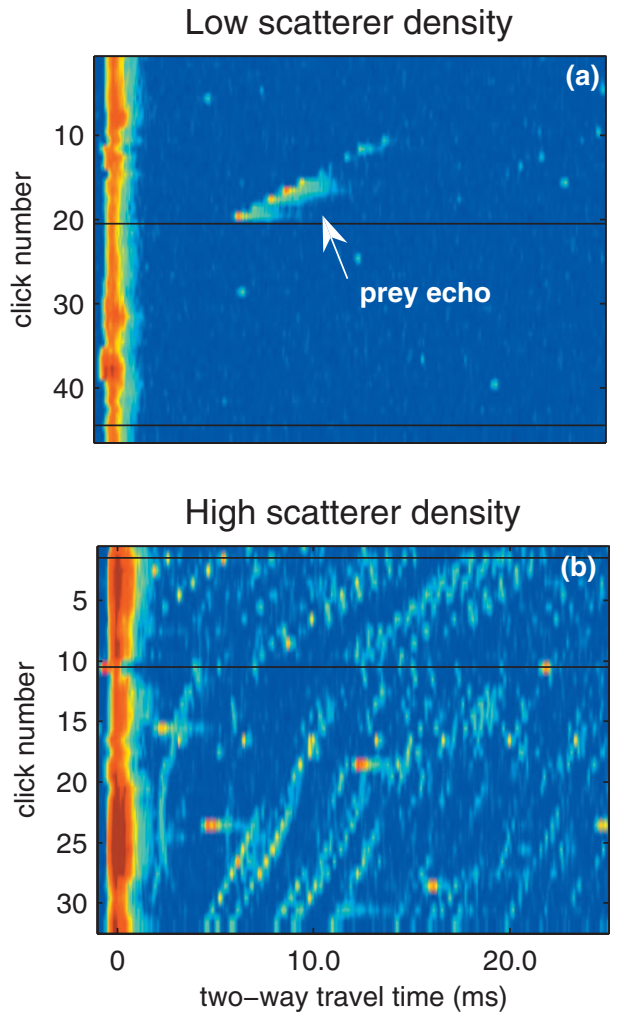

FIG. 2. Two representative examples of echograms displaying scattered FM clicks of a foraging beaked whale which are used to identify echo trains: (a) low density of scatterers, (b) high density of scatterers. Click number is shown on the vertical axis and the horizontal axis shows time since last emitted click. Echo strength is indicated by color with red corresponding to higher sound pressure and blue corresponding to lower sound pressure. Plot (a) shows a single echo train as the whale approaches a target. The horizontal black line represents a period of greater than $1 \mathrm{~s}$ between outgoing clicks that meet a preset threshold level which, in this case, indicates the whale's switch to the lower amplitude buzz.

\section{Identification of buzzes, echoes and echo trains}

This analysis was restricted to the section of the tag recording made during the three foraging dives in which the tag was dorsally located. A supervised, click detection algorithm was used to identify FM clicks and buzzes (Johnson et al., 2006). Echograms were then constructed from the sequences of FM clicks produced by the tagged whale (see Fig. 2) by aligning the envelopes of $25 \mathrm{~ms}$ sections of the tag recording starting at each click (Johnson, 2004). The duration of the sections restricts the maximum two-way travel time (TWTT) of echoes to $25 \mathrm{~ms}$ corresponding to a maximum range from source to scatterer of $19 \mathrm{~m}$. This TWTT limit ensures adequate echo-to-noise ratio, ENR, for later processing. Echo trains from a single scatterer were readily identifiable in the echograms as series of echoes with consistent, slowly varying TWTT (Fig. 2(a)).

Segments of the tag sound recording with a duration of $400 \mu \mathrm{s}$, centered on each echo from an individual target, were extracted for analysis. This window size was selected to accommodate the increased duration of echoes of FM clicks caused by scattering from a finite-sized object. Another $400 \mu$ s segment was taken $600 \mu$ s prior to each echo as a contemporary sample of the noise levels. The signal and noise segments were filtered with a 12-pole (six low-pass 
and six high-pass) Butterworth bandpass filter with cutoff frequencies of 20 and $70 \mathrm{kHz}$ to reduce out-of-band noise. The echo level, $E L$, and the noise level, $N L$, were estimated as $10 \log _{10}($.$) of the signal power in each echo and noise$ segment, respectively. The ENR was then estimated from the difference $E L-N L$. The magnitude of the spectrum of each echo was estimated by calculating a 512-bin fast Fourier transform (FFT).

\section{Whale-selected and nonselected targets}

Echo trains were grouped according to whether they occurred in the proximity of a buzz, indicating that the whale tried to capture the target, or not. Echo trains that terminated within $5 \mathrm{~s}$ of a buzz were considered to represent a whaleselected target (Madsen et al., 2005). If multiple echo trains were detectable prior to a buzz, indicating multiple scatterers, the echogram was expanded in the vicinity of the buzz to look for echoes associated with low-level buzz clicks. If an echo train associated with the buzz was detected, then the echo train immediately prior to the buzz that most closely matched the TWTT of the echoes associated with the buzz was chosen as the whale-selected target. However, if no echo was visible from the buzz clicks, or two echo trains were very close together in time prior to the buzz, that sequence was not considered in the analysis. An echo observed during the buzz, though, was not a criterion for a whale-selected target if there was only one echo train leading up to the buzz. Echo trains occurring more than $5 \mathrm{~s}$ prior to a buzz were considered to represent targets not selected by the whale. A subset of these echo trains was randomly chosen for comparison using the same maximum TWTT criteria as for whale-selected echo trains.

\section{Spectral classification}

Acoustic scattering spectra of marine organisms are characterized by interference patterns specific to the size, shape, material properties, and orientation of the organism. This information should be contained in the echoes recorded by the DTAG; however, the echo spectrum will also be influenced by the spectrum of the sound source and any interfering noise. While the effects of noise can be minimized by selecting echoes with a high ENR, the spectrum of the sound source will depend on the unknown angle of the scatterer to the axis of the outgoing sonar click. Given the broad, smooth spectrum of on-axis FM clicks, targets close to the acoustic axis should give rise to echoes with spectral features associated only with the scattering characteristics of the target. In contrast, off-axis targets will produce echoes with features due to both the target and off-axis distortion.

Assuming M. densirostris have a beam pattern similar to Z. cavirostris and delphinids, the output energy of the FM clicks will decrease rapidly with increasing off-axis angle outside of the main beam (Zimmer et al., 2005). Thus, by placing a minimum ENR criterion on echoes chosen for analysis a set can be obtained with a preponderance of echoes from on-axis targets.

Given the limited ENR of echoes available in this study, three robust parameters were chosen to characterize the echo

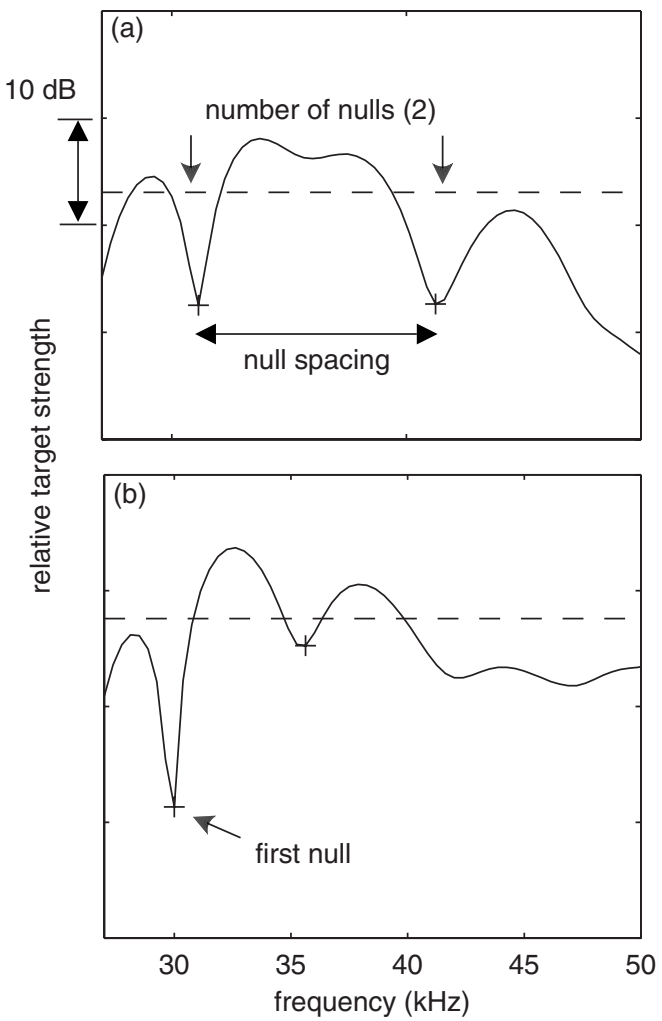

FIG. 3. Example of automated structure analysis results. Plus signs indicate nulls and horizontal dashed lines indicate mean relative target strength of frequency band displayed. An example of the two criteria for a null are illustrated in the plot (b): (1) The dip near $42 \mathrm{kHz}$ does not meet the criterion of being more than $1 \mathrm{~dB}$ lower than both adjacent peaks; (2) the dip at $47 \mathrm{kHz}$ does not meet the criterion of being more than $4 \mathrm{~dB}$ lower than the average of the two adjacent peaks.

spectra: (1) number of nulls, (2) frequency of the first null, and (3) frequency interval between the first two nulls (Fig. $3)$. Nulls were defined as any dip in the spectral magnitude with greater than $4 \mathrm{~dB}$ difference between the local minimum and the average of the two adjacent maxima. To avoid detecting small ripples in the spectrum, possibly due to noise, nulls were rejected if either adjacent peak was less than $1 \mathrm{~dB}$ above the null. The frequency band between 27 and $50 \mathrm{kHz}$ was selected for analysis. This range falls within the $-10 \mathrm{~dB}$ end points of the FM click signal and is also within the range of frequencies over which on-axis FM clicks appear to be largely featureless (Fig. 1(b)). To verify that on-axis clicks from $M$. densirostris are indeed featureless in this band, we counted the number of spectral nulls in a set of 144 likely on-axis clicks from a conspecific measured by Johnson et al. (2006). According to the definition given above, only six $(<5 \%)$ of these clicks had one or more nulls supporting the notion that on-axis FM clicks are essentially featureless.

A Monte Carlo simulation, using 1000 trials, was performed by combining an on-axis $M$. densirostris click with artificial, band-limited $(20-70 \mathrm{kHz})$ noise to determine the likelihood that noise in the simulated echo signal would give rise to spectral nulls. With an in-band ENR of $15 \mathrm{~dB}$, fewer than $2 \%$ of the trials (14 of 1000) resulted in a spectral null of at least $4 \mathrm{~dB}$ in depth in the frequency range of interest $(27-50 \mathrm{kHz})$. No simulated echo had more than one spectral 
null. Thus, by selecting echoes with ENR $>15 \mathrm{~dB}$, nulls in the resulting echo spectra are most likely due to the scattering characteristics of the target, as opposed to off-axis distortion or noise.

\section{Target strength estimation}

Target strength, $T S$, can be calculated from the active sonar equation (Urick, 1983):

$$
T S=E L+2 T L-S L,
$$

where $T L$ and $S L$ are the one-way transmission loss and source level, respectively, in decibels. The $T L$ for spherical spreading is given by $T L=20 \log r+\alpha r$, where $r$ is the oneway distance from the source to the scatterer and $\alpha$ is the absorption loss in $\mathrm{dB} / \mathrm{m}$. Absorption can be neglected as its effect on $T L$ for short target distances is small $(<0.5 \mathrm{~dB})$ in the frequency range considered here. All relative target strengths presented in this study are band averaged, in which the mean is calculated prior to logarithmic conversion.

Although $E L$ and $T L$ can be estimated from the tag recordings, the $S L$ of $M$. densirostris is not precisely known and may vary from click to click. The location of the tag behind the whale's head, in the near field and off axis with respect to the sound source, prevents measurements of $S L$. However, measurements of the outgoing FM clicks by the tag provide a proxy for changes in the power output of the on-axis emissions from click to click (Madsen et al., 2005). Thus, the relative $T S$ (i.e., the $T S$ with respect to an unknown source level) can be estimated by replacing $S L$ by $S_{h}$, the received level in decibels of the outgoing click as recorded by the tag. The relative $T S$, although not useful in an analysis requiring absolute measures of target strength, can be used in quantitative comparisons between echoes observed at a given tag position on the same whale.

\section{Statistical comparison of echo trains}

A statistical comparison of echo parameters across individual echoes is complicated by the fact that echoes within the same echo train represent the same target, and so are not independent samples. To overcome this problem, we use echo trains as the unit of analysis and combine the parameters of echoes within the train into a summary statistic. For each parameter (e.g., number of nulls), a set of discrete levels or bins was selected. The proportion of the echoes in each echo train with a parameter value falling in each bin was calculated producing a histogram of that parameter for each echo train. This reduces the variable length echo trains into fixed-length vectors suitable for statistical analysis. All histograms were normalized by the number of echoes in each echo train. As an example of this technique, consider an echo train containing four echoes with 3,2, 0, and 3 spectral nulls in the echoes. If the null-count parameter was represented by bins of $0,1,2, \ldots$ nulls, the histogram for this train would be

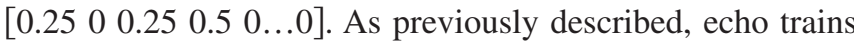
were differentiated according to whether they occurred close to a buzz or not (i.e., whether the whale appeared to select the target as a prey item or not). Thus, two sets of histograms were generated summarizing the echoes in whale-selected and nonselected targets.

\section{Density of scatterers}

In addition to the relative TS and spectral content of echoes, the depth and density of scatterers were also estimated from the data. Scatterer density was inferred from the number of echoes visually detected in the echogram within a 25 ms window following each FM click (Fig. 2). The number of echoes per click, in the proximity of buzzes, had a strongly bimodal distribution with either few (1-5) echoes or many $(>10)$ echoes being excited by each click. Only three of the 47 buzzes analyzed occurred in an environment of intermediate scatterer density (i.e., with between five and ten echoes recorded for each FM click prior to the buzz). Based on this, we define intervals in the data in which there were more than five echoes per click as having high scatterer density and the converse for low scatterer density.

\section{RESULTS}

During dives 2 through 4, the dives during which the tag was located near the dorsal ridge, the whale dove to depths between 600 and $1320 \mathrm{~m}$. FM clicks were only observed at depths greater than $440 \mathrm{~m}$. As indicated by buzzes observed during these dives, the whale hunted at various meso- and bentho-pelagic depths. During dive 3 the whale hunted near the seafloor at depths between 580 and $680 \mathrm{~m}$. Foraging during dives 2 and 4 appeared to be spread out in depth over several hundred meters; although, in both cases the majority of buzzes were concentrated in depth ranges of less than $200 \mathrm{~m}$.

\section{A. Echoes selected for analysis}

A total of 89 buzzes were observed during the three dives $(34,24$, and 31 in dives 2,3 , and 4, respectively) indicating prey capture attempts. Of these, 47 were preceded by unambiguous echo trains that are assumed to correspond to scattering from the selected prey. The remaining buzzes were either associated with irresolvable echo trains due to a cluttered acoustic environment or not associated with echo trains with sufficient ENR. In dive 3, a high percentage of buzzes could not be correlated with echo trains due to reverberation from the nearby seafloor. In general, when an echo train was associated with a buzz, the first echo of the train was discernable from background noise at a distance from the tag to the scatterer of between 5 and $15 \mathrm{~m}$. In each case the echo train terminated shortly before the start of the buzz at a distance of 3-5 $\mathrm{m}$.

The 47 echo trains identified as corresponding to whaleselected prey contained a total of 426 discrete echoes. Of that total, 132 echoes, in 37 echo trains, met the minimum ENR criterion. In order to accumulate a sufficient number of echoes for comparison, 92 echo trains from scatterers not selected by the whale were chosen at random. Of these, 42 trains containing 120 echoes remained after applying the same ENR criterion. The number of echoes analyzed from each dive is shown in Table I. Many more nonselected echo 
TABLE I. Distribution of echo trains (bold) and echoes (parenthesis) from whale-selected and nonselected scatterers observed in three dives examined. All echoes selected for analysis (bottom half of table) had an echo-to-noise ratio, ENR, of at least $15 \mathrm{~dB}$.

\begin{tabular}{lcc}
\hline \hline & Whale selected & Nonselected \\
\hline Total identified & & \\
Dive 2 & $\mathbf{1 8}(139)$ & $\mathbf{3 6}(276)$ \\
Dive 3 & $\mathbf{9}(77)$ & $\mathbf{1 8}(130)$ \\
Dive 4 & $\mathbf{2 0}(209)$ & $\mathbf{3 8}(177)$ \\
Total & $\mathbf{4 7}(425)$ & $\mathbf{9 2}(583)$ \\
Selected for analysis & & \\
Dive 2 & $\mathbf{1 4}(43)$ & $\mathbf{2 2}(67)$ \\
Dive 3 & $\mathbf{5}(18)$ & $\mathbf{1 2}(37)$ \\
Dive 4 & $\mathbf{1 8}(71)$ & $\mathbf{8}(16)$ \\
Total & $\mathbf{3 7}(132)$ & $\mathbf{4 2}(120)$ \\
\hline \hline
\end{tabular}

trains were required to obtain a sample size similar to that of the whale-selected echo trains as they generally had fewer echoes in each train. This is likely due to the shorter length of time that the whale maintained these nonselected scatterers within its acoustic beam.

\section{B. Echo classification}

\section{Spectral characteristics}

The spectra of most individual echoes from prey selected by the whale contained one or more nulls within the frequency band examined (e.g., Fig. 4). Additionally, echoto-echo variability was observed in the spectra of some echo trains (e.g., Figs. 4(a) and 4(b)). Echoes from whale-selected targets had more spectral nulls than did echoes from scatterers not selected by the whale (median: whale-selected: 2, nonselected:1; Wilcoxon rank-sum $p=0, n=79)$. Notably, less than $12 \%$ of the echoes within whale-selected echo trains were characterized by featureless echoes (i.e., echoes with no nulls meeting the criteria defined above), whereas more than $48 \%$ of the echoes within nonselected echo trains were composed of such echoes (Fig. 5(a)).

A comparison of other spectral features between the whale-selected and nonselected echo trains show less notable differences. No significant differences were found in the medians of the first null locations and the spacings between the first two nulls (Figs. 5(b) and 5(c)) between whale-selected and nonselected targets [(Wilcoxon rank-sum $p=0.11, n$ $=64$ (null location), $p=0.14, n=45$ (null spacing) .

\section{Target strengths}

Echo trains of targets selected by the whale were composed of echoes with significantly higher relative target strengths [median difference of $12 \mathrm{~dB}$, Wilcoxon rank-sum: $p=0, n=79$ (Fig. $5(\mathrm{~d}))]$ than nonselected targets. Echo trains from whale-selected prey also showed relatively wide echoto-echo variation in relative target strengths. For echo trains containing at least two echoes, $73 \%$ contained echoes that varied by at least $3 \mathrm{~dB}$ and $40 \%$ contained echoes varying by at least $6 \mathrm{~dB}$. Echo trains of targets not selected by the whale had less variability with $55 \%$ of echo trains having at least a $3 \mathrm{~dB}$ variation and none varying by more than $6 \mathrm{~dB}$. This difference in variability between echo trains of targets se- lected by the whale and those not selected may be due, in part, to the fact that there are fewer echoes, on average, in the echo trains of nonselected targets.
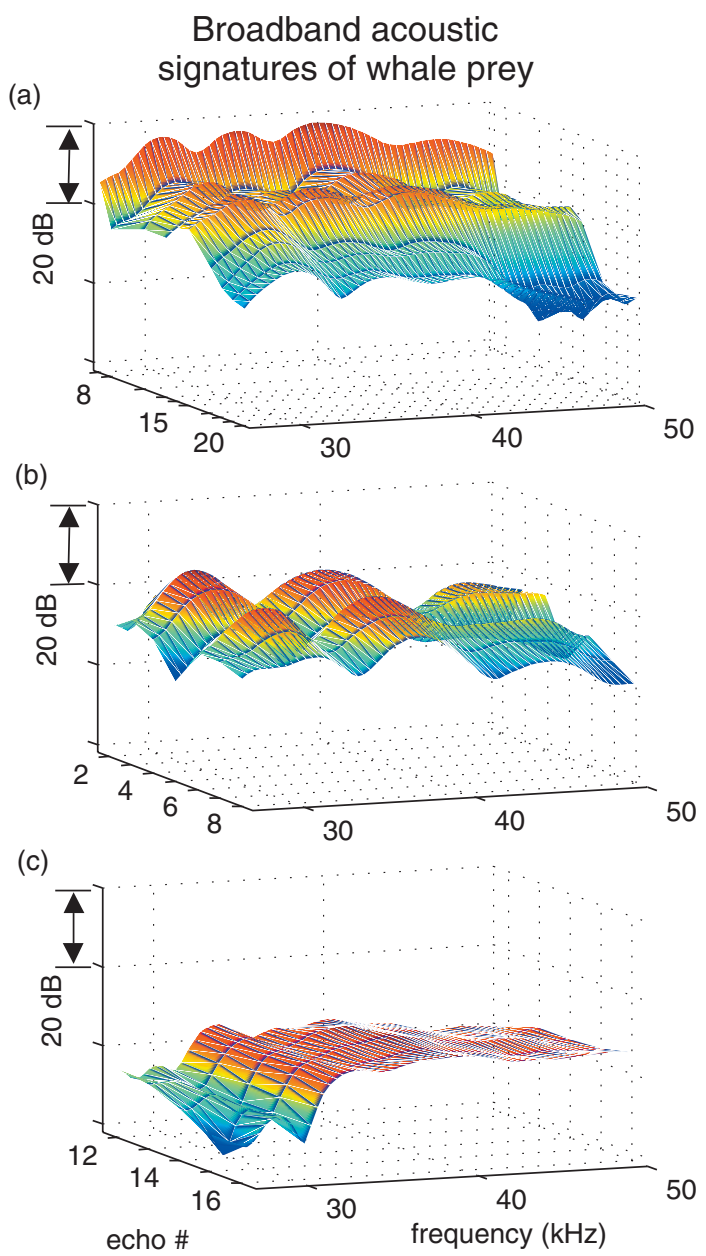

FIG. 4. Broadband acoustic signatures of three prey selected by the whale. Each plot is comprised of frequency spectra of a series of echoes that make up one echo train. Only echoes meeting the ENR criterion are included, hence not all echoes in each echo train are displayed. The echo number of displayed echoes are indicated by the presence of a tick mark. Echo values are interpolated across the gaps associated with echoes not chosen such as between echoes 15 and 17 in plot (a). Plots (a) and (b) show examples of high target strength prey observed at deep depths (below $700 \mathrm{~m}$ ) in environments with low scatterer density. Plot (c) shows an example of lower target strength prey observed in shallower water (above $700 \mathrm{~m}$ ) in environments with high scatterer density. 


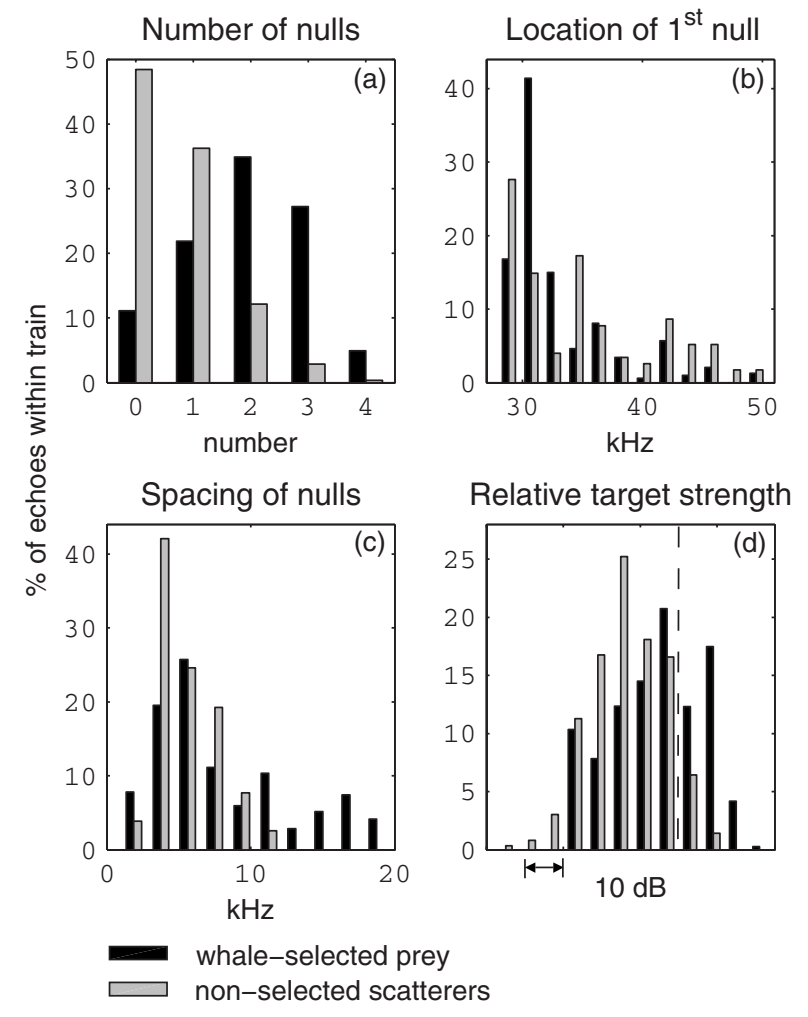

FIG. 5. Normalized distributions of various characteristics of echoes from whale-selected prey and randomly chosen scatterers not selected by the whale (black and gray bars, respectively). The characteristics are: (a) number of nulls, (b) location of first null, (c) spacing between the first two nulls, and (d) relative target strength. Each characteristic of an individual echo is weighted by the fraction of the total number of echoes within a train that the echo represents. Distributions are normalized by the total number of echo trains (whale selected or nonselected). Vertical dashed line in target strength plot (d) represents absolute target strength value of $-65 \mathrm{~dB}$ based on an arbitrary source level of $200 \mathrm{~dB}$.

\section{Comparison of echo characteristics between two groups of prey}

Scatterer density in regions where the whale hunted varied widely within the three dives (Fig. 2) and two distinct regimes can be identified. Scatterer density was low during much of dives 2 and 4 while high scatterer density was observed throughout the shallower and benthic dive 3 (Fig. 6). The two density regimes were separated spatially in the water column with the high density group found, generally, in water shallower than $700 \mathrm{~m}$ and the lower density group found at greater depths. A comparison was made between these two groups in which the three outliers (i.e., scatterers in high density environments, deeper than $700 \mathrm{~m}$ ) were discounted.

In both regimes, the targets selected by the whale were characterized by more highly structured echoes than the group of nonselected scatterers. In the low scatterer density case (Fig. 7(b)) the median number of nulls for whaleselected and nonselected targets were two and one, respectively (Wilcoxon rank-sum $p=0, n=52$ ). Similarly, in the high scatterer density case (Fig. 7(a)), the median values were one and zero, respectively (Wilcoxon rank-sum $p$ $=0.04, n=27$ ); although, the result in the high density regime is not significant if Bonferroni correction is taken into account on the division of data. As in the combined results, a

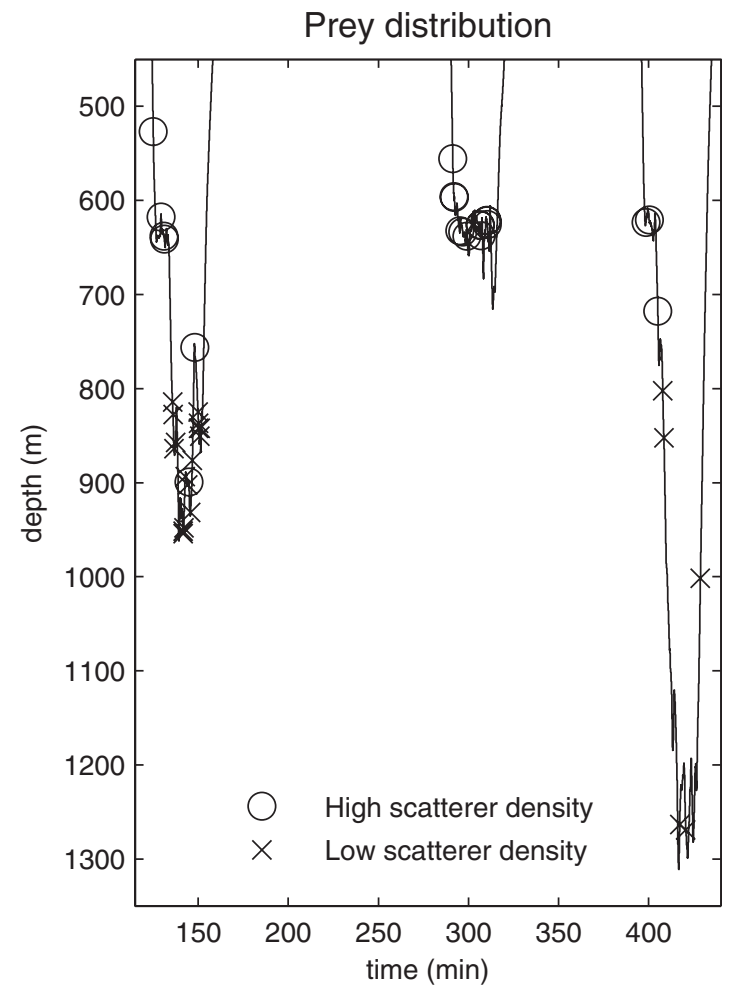

FIG. 6. Depth distribution of prey categorized by scatterer density. Depth is truncated above $450 \mathrm{~m}$ as no predation events were observed at shallower depths.

relatively small percentage of echoes in whale-selected echo trains were composed of featureless echoes in both the shallow, high density group (whale selected: $14 \%$, versus nonselected: $57 \%$ ) and the deep, low density group (whale selected: $11 \%$, nonselected: 40\%). Finally, a comparison between the two environments (high and low scatterer density) of only whale-selected targets showed no significant difference in the number of nulls.

The distribution of relative target strengths of the two populations provided further information about these two scattering groups. The target strength distribution of whaleselected and nonselected echoes within each environment (i.e., shallow, high scatterer density and deep, low scatterer density) are overlapping (Fig. 8) with no significant difference noted in the median target strengths in the shallow, high scatterer density case (Wilcoxon rank-sum $p=0.36, n=27$ ). A difference of $6 \mathrm{~dB}$ in the medians was observed in the deep, low scatterer density case with whale-selected targets having higher target strengths $(p=0.02, n=52)$. Furthermore, a comparison between the two environments (i.e., high and low echo density regimes) of only the whale-selected targets reveals a $15 \mathrm{~dB}$ difference in the median target strengths with the targets in the deep, low density regime having higher values $(p=0, n=37)$.

In order to explore the implication of this difference in target strengths, a scattering model was used to estimate the possible difference in average scatterer size. Using a simple, finite cylinder model for randomly oriented scatterers of length, $L$, target strength averaged over orientation varies as $10 \log \left(L^{2}\right)$ in the geometric scattering region (Stanton et al., 1993). This first-order approximation can be used to estimate 


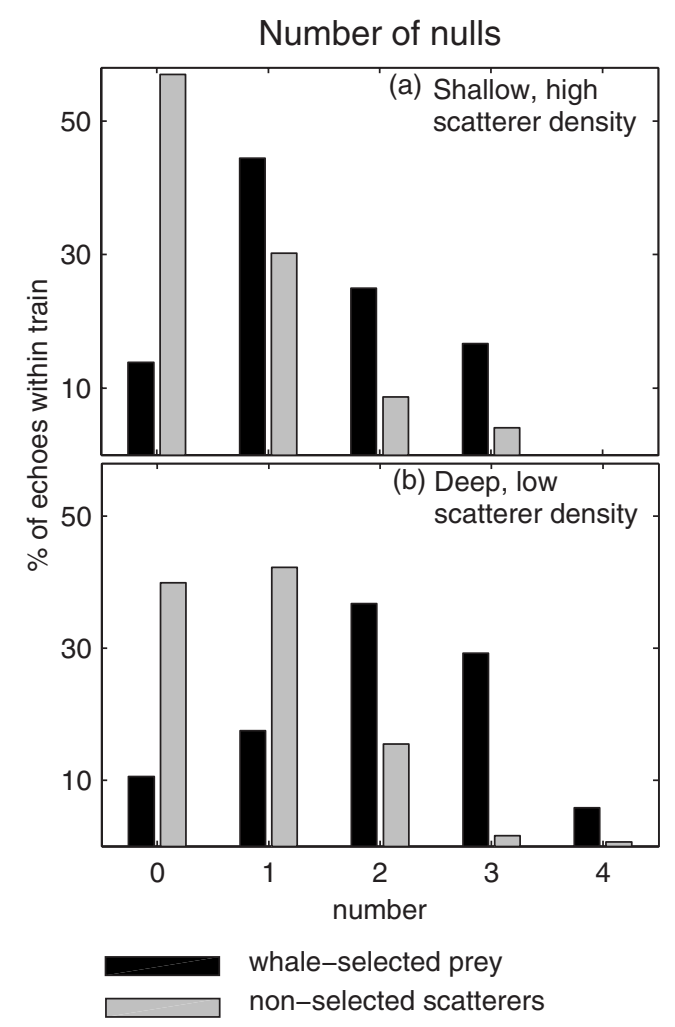

FIG. 7. Normalized distributions of number of nulls in frequency responses of selected and nonselected scatterers (black and gray bars, respectively) in (a) shallow, high density aggregations and (b) deep, low density aggregations. Weighting and normalization are identical to Fig. 5.

a ratio of the lengths of prey from the difference observed in average target strengths. For scattering from two organisms of the same shape and material composition, a $15 \mathrm{~dB}$ difference in target strengths correlates to a length factor of approximately 5.5 .

\section{DISCUSSION AND CONCLUSIONS}

The characteristics of echoes from targets ensonified by broadband echolocation signals have been studied through data obtained, in situ, by a recording device mounted on a foraging Blainville's beaked whale. By setting stringent criteria on the echoes analyzed and, in part, due to the opportunely smooth spectrum of the whale's emitted signal, the spectral characteristics of backscattering by prey have been analyzed and relative target strengths have been estimated.

Significant structure, resembling the type of interference patterns observed when marine organisms scatter broadband sound in laboratory experiments exist in the frequency spectra of echoes measured in this study. Additionally, echo-toecho variability in terms of both spectral structure and relative target strengths is observed within some echo trains selected by the whale. Although both spectral distortion of the emitted signal, related to the prey location within the acoustic beam, and noise can induce spectral nulls in the received echoes, these effects have been minimized by selecting only high ENR echoes. Therefore, variations in echo characteristics within echo trains, arguably, relate to the orientation of the scatterer with respect to the incident sound beam rather than location in the beam. Other modeling stud-

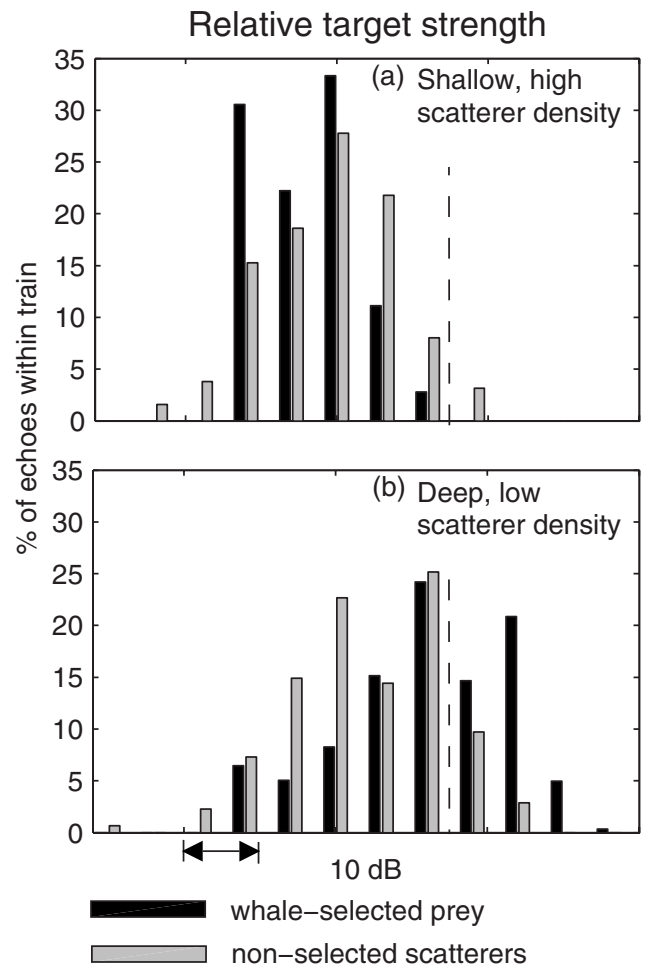

FIG. 8. Normalized distributions of relative target strengths of whaleselected prey and nonselected scatterers (black and gray bars; respectively) in: (a) shallow, high density aggregations, and (b) deep, low density aggregations. Vertical dashed line represents absolute target strength value of $-65 \mathrm{~dB}$ based on an arbitrary source level of $200 \mathrm{~dB}$. Weighting and normalization are identical to Fig. 5.

ies and laboratory experiments of the broadband spectra of echoes from fish and squid show a qualitatively similar variability (Reeder et al., 2004; Jones, 2006). In these studies of scattering from individual organisms, changes in orientation as small as $5^{\circ}$ have been shown to dramatically change the backscattering spectra.

Comparing all groups of echoes from scatterers selected by the whale to other randomly chosen, nonselected scatterers, we have shown that the targets preyed upon by the whale have a higher degree of structure within their frequency responses. Conversely, the whale was much less likely to prey upon targets characterized by featureless echoes. This result is contrary to what would be expected if structure in the echoes resulted primarily from the location of the scatterer with respect to the axis of the sonar beam. There is no behavioral reason to suppose that whale-selected targets would tend to be ensonified further off-axis than nonselected targets. In fact, the contrary ought to be the case as is seen in bats (Moss and Ghose, 2003), i.e., the whale likely orients itself so as to ensonify selected targets close to its acoustic axis. Thus, neglecting the frequency response of the scatterer, one would expect to see more spectral nulls in the nonselected targets than in those selected for capture attempts. Here we see a significant trend in the opposite direction indicating that the observed spectral features are most likely due to the frequency-dependent target strength of the scatterer.

Given our lack of knowledge of the type, size, and orientation of the ensonified scatterers, no definite statement 
can be made regarding the type or size of organisms that might produce the structured versus featureless echoes, or the reason for the whale preferring more structured returns. It is even possible that the whale's decision process is completed at a further range than that of the targets considered in this study. It must be assumed that the whale's range of detection is greater than that of the tag-mounted hydrophones. The combination of directivity of the whale's aural receiving system, as shown by Au and Moore (1984) in other toothed whales, and poorer ENR of the hydrophone outputs due to flow noise over the tag should provide the whale with a significantly better ENR. Nonetheless, it is clear from this study that there is a significant difference in the echo characteristics of prey selected by the whale and those not selected when within the range of our sensor. Possible explanations for increased structure in the selected scatterers are that they are a different species, different size, and/or in a different orientation when ensonified. Stomach content data, net samples, and acoustic scattering studies of the species present in the areas and depths at which Blainville's beaked whales forage are needed to narrow down the type and size of organism giving rise to the echoes, and to ultimately allow the results to be interpreted at an ecological level.

Two depth-stratified regimes were identified which exhibited differences in scatterer density and prey target strength. The whale hunted prey with lower target strengths in high density aggregations between depths of 600 and $650 \mathrm{~m}$. Prey with higher target strengths were selected by the whale at depths below $700 \mathrm{~m}$ in environments with low scatterer density. In the shallow, high scatterer density environment the target strength distributions of selected and nonselected targets showed no significant difference suggesting that the relative target strength of a scatterer is not the whale's sole means of discriminating between prey and nonprey targets. In the low scatterer density environment it is clear that the whale favored targets with a significantly higher degree of structure in their echo spectra. Combined, these results provide some evidence of spectral-based prey selection, though further data will be required to determine whether this is the whale's primary method of discrimination.

Finally, an explanation has been proposed as to why this large marine predator chooses to dive to significantly deeper depths, and thus expend greater amounts of energy (Tyack et al., 2006), to hunt less dense populations of organisms. A significant difference in the relative target strengths for whale-selected targets was observed between the shallow, high density aggregation and the deep, low density aggregation. While several parameters can give rise to a difference in target strengths (e.g., size, shape, tissue composition and gas inclusions), we have shown that, by allowing length to dominate, the higher target strengths observed at deeper depths could be explained by a prey size some 5-6 times that of prey hunted in shallower water. This, in turn, could give the whale an incentive to expend the energy required to dive to greater depths.

Little is known about the potential prey communities in the deep ocean due to the difficulty and expense of studying these habitats. The method presented here, using a deep- diving, echolocating predator as a natural source of sound and analyzing the scattered signals, is a novel way to explore this challenging environment. It is hoped that future studies will provide data which can be used to further analyze these whales' behavior and characterize the prey field where they hunt.

\section{ACKNOWLEDGMENTS}

Thanks to Natacha Aguilar de Soto and the El Hierro field team of the University of La Laguna for data collection. Thanks also to Tom Hurst and Alex Shorter for DTAG construction. Field work was supported by the U.S. National Oceanographic Partnership Program, the U.S. Office of Naval Research, and the Canary Islands government. Research was conducted under a permit from the government of the Canary Islands to the University of La Laguna and was approved by the WHOI Institutional Animal Care and Use Committee. Analysis of the data was supported by the Office of the Oceanographer of the U.S. Navy, The Academic Programs Office at the Woods Hole Oceanographic Institution and the Danish Natural Science Research Council through a Steno scholarship to Peter T. Madsen.

Au, W. W. L. (1993). The Sonar of Dolphins (Springer-Verlag, New York). Au, W. W. L., and Benoit-Bird, K. J. (2003). "Acoustic backscattering by Hawaiian lutjanid snappers. II. Broadband temporal and spectral structure," J. Acoust. Soc. Am. 114, 2767-2774.

Au, W. W. L., and Moore, P. W. B. (1984). "Receiving beam patterns and directivity indices of the Atlantic bottlenose dolphin Tursiops truncatus," J. Acoust. Soc. Am. 75, 255-262.

Au, W. W. L., and Pawloski, J. L. (1989). "Detection of noise with rippled spectra by the Atlantic bottlenose dolphin," J. Acoust. Soc. Am. 86, 591596.

Dubrovskiy, N. (1989). "On the two auditory subsystems in dolphins," in Proceedings of a NATO Advanced Workshop and Symposium of the Fifth International Theriological Congress on Sensory Abilities of Cetaceans, edited by R. Thomas and J. A. Kastelein, NATO ASI Series (Plenum, Rome, 1990), pp. 233-254.

Fuzessery, Z., Feng, A., and Supin, A. (2004). "Central auditory processing of temporal information in bats and dolphins," in Echolocation in Bats and Dolphins, edited by C. F. V. M. Thomas and J. A. Moss (University of Chicago Press, Chicago), pp. 115-122.

Griffin, D. R. (1958). Listening in the Dark; the Acoustic Orientation of Bats and Men (Yale University Press, New Haven).

Johnson, M., Madsen, P., Zimmer, W., Aquilar-Soto, N., and Tyack, P. (2006). "Foraging Blainville's beaked whales (Mesoplodon densirostris) produce distinct click types matched to different phases of echolocation," J. Exp. Biol. 209, 5038-5050.

Johnson, M., Madsen, P. T., Zimmer, W. M. X., de Soto, N. A., and Tyack, P. L. (2004). "Beaked whales echolocate on prey," Proc. R. Soc. London, Ser. B 271, S383-S386.

Johnson, M. P., and Tyack, P. L. (2003). "A digital acoustic recording tag for measuring the response of wild marine mammals to sound," IEEE J. Ocean. Eng., 28, No. 1, 3-12.

Jones, B. A. (2006). "Acoustic scattering of broadband echolocation signals from prey of Blainville's beaked whales: Modeling and analysis," Master of Science, Massachusetts Institute of Technology and Woods Hole Oceanographic Institution.

MacLeod, C. D., Santos, M. B., Lopez, A., and Pierce, G. J. (2006). "Relative prey size consumption in toothed whales: Implications for prey selection and level of specialization," Mar. Ecol.: Prog. Ser. 326, 295-307.

Madsen, P. T., Johnson, M., de soto, N., Zimmer, W. M. X., and Tyack, P. (2005). "Biosonar performance of foraging beaked whales (Mesoplodon densirostris)," J. Exp. Biol. 208, 181-194.

Madsen, P. T., Wahlberg, M., and Mohl, B. (2002). "Male sperm whale (Physeter macrocephalus) acoustics in a high-latitude habitat: Implications for echolocation and communication," Behav. Ecol. Sociobiol. 53, 31-41. Martin, L. V., Stanton, T. K., Wiebe, P. H., and Lynch, J. F. (1996). "Acous- 
tic classification of zooplankton," ICES J. Mar. Sci. 53, 217-224.

Martin Traykovski, L. V., Stanton, T. K., Wiebe, P. H., and Lynch, J. F. (1998). "Model-based covariance mean variance classification techniques: Algorithm development and application to the acoustic classification of zooplankton,” IEEE J. Ocean. Eng. 23, 344-363.

Miller, P. J. O., Johnson, M., and Tyack, P. L. (2004). "Sperm whale behaviour indicates the use of echolocation click buzzes 'creaks' in prey capture," Proc. R. Soc. London, Ser. B 271, 2239-2247.

Moss, C. F., and Ghose, K. (2003). "The sonar beam pattern of a flying bat as it tracks tethered insects," J. Acoust. Soc. Am. 114, 1120-1131.

Nachtigall, P. E. (1980). "Odontocete echolocation performance on object size, shape, and material: in animal sonar systems," in Proceedings of the Second International Interdisciplinary Symposium on Animal Sonar Systems, edited by J. Busnel and R. G. Fish (Plenum, Jersey, Channel Islands), pp. 71-95.

Pauly, D., Trites, A. W., Capuli, E., and Christensen, V. (1998). "Diet composition and trophic levels of marine mammals," ICES J. Mar. Sci. 55, 467-481.

Reeder, D. B., Jech, J. M., and Stanton, T. K. (2004). "Broadband acoustic backscatter and high-resolution morphology of fish: Measurement and modeling," J. Acoust. Soc. Am. 116, 747-761.

Simmonds, E. J., Armstrong, F., and Copland, P. J. (1996). "Species identification using wideband backscatter with neural network and discriminant analysis,” ICES J. Mar. Sci. 53, 189-195.
Simmonds, E. J., and Maclennan, D. N. (2005). Fisheries Acoustics, Fish and Fisheries (Blackwell Science Ltd., Oxford).

Stanton, T. K., Chu, D., Wiebe, P. H., and Clay, C. S. (1993). "Average echoes from randomly oriented random-length finite cylinders: Zooplankton models," J. Acoust. Soc. Am. 94, 3463-3472.

Stanton, T. K., Chu, D., Wiebe, P. H., Martin, L. V., and Eastwood, R. L. (1998). "Sound scattering by several zooplankton groups. I: Experimental determination of dominant scattering mechanisms," J. Acoust. Soc. Am. 103, 225-235.

Tyack, P., Johnson, M., Aquilar-Soto, N., Sturlese, A., and Madsen, P. T. (2006). "Extreme diving of beaked whales," J. Exp. Biol. 209, 4238-4253.

Urick, R. J. (1983). Principles of Underwater Sound, 3rd edition (McGrawHill, New York).

Vel'min, V. A., and Dubrovskiy, N. A. (1975). "Auditory analysis of sound pulses in dolphins (in Russian)," Proceedings of the Academy of Sciences of USSR 225, 470-473.

Whitehead, H., MacLeod, C. D., and Rodhouse, P. (2003). "Differences in niche breadth among some teuthivorous mesopelagic marine mammals," Marine Mammal Sci. 19, 400-406.

Zakharia, M. E., Magand, F., Hetroit, F., and Diner, N. (1996). "Wideband sounder for fish species identification at sea," ICES J. Mar. Sci. 53, 203208 .

Zimmer, W. M. X., Johnson, M. P., Madsen, P. T., and Tyack, P. L. (2005). "Echolocation clicks of free-ranging Cuvier's beaked whales (Ziphius cavirostris)," J. Acoust. Soc. Am. 117, 3919-3927. 